\title{
A GENERALIZED CROSS-PROPERTY RELATION BETWEEN THE ELASTIC MODULI AND CONDUCTIVITY OF ISOTROPIC POROUS MATERIALS WITH SPHEROIDAL PORES
}

\author{
"WILLI PABST, EVA GREGOROVÁ \\ Department of Glass and Ceramics, University of Chemistry and Technology, Prague (UCT Prague) \\ Technická 5, 16628 Prague 6, Czech Republic \\ "E-mail: willi.pabst@vscht.cz
}

Submitted September 26, 2016; accepted November 16, 2016

\begin{abstract}
Keywords: Elasticity, Conductivity (thermal, electrical), Porosity, Pore shape (prolate, oblate, isometric, anisometric, spherical, spheroidal), Pore channels, Microcracks, Aspect ratio, Poisson ratio (Poisson number, non-auxetic), Elastic moduli (Young's modulus, tensile modulus, shear modulus, bulk modulus, compressive modulus), Cross-property relation, EshelbyWu coefficient, Maxwell coefficient

A new generalized cross-property relation is proposed for predicting the relative elastic moduli (Young's modulus, shear modulus, bulk modulus) from the relative conductivities (thermal or electrical) of isotropic porous materials with spheroidal pores. Using this cross-property-relation it is possible to estimate the elastic moduli when the conductivites are known (either from real-world measurements or from numerical calculations on digital microstructures) and vice versa. This generalized cross-property relation contains the case of spherical or isometric pores as a special case, but is sufficiently general to account for the properties of materials with strongly anisometric pores, i.e. randomly orientated prolate and oblate pores, including the extreme cases of pore channels or microcracks. The exponent of this cross-property relation is shown in graphical form and-for future reference with respect to practical applications - its numerical values are listed in tabular form as a function of the pore aspect ratio and the Poisson ratio of the solid.
\end{abstract}

\section{INTRODUCTION}

The prediction of the effective properties of porous materials is a long-standing problem of materials science and is still of broad current interest [1-6]. From the practical point of view it is often desirable to predict, or at least roughly estimate, the relative property of a porous material, i.e. the non-dimensional ratio of its effective property and the property of the dense solid, based on porosity information alone, i.e. only on the basis of knowing the volume fraction of pores. An exact prediction is possible only for certain special cases, e.g. for the axial component of the (thermal or electrical) conductivity or Young's modulus of certain anisotropic microstructures, namely translationally invariant ones, or for dilute systems with spherical pores, but for most microstructures of practical interest only upper bounds (Voigt bound, upper Wiener bound, upper Hashin-Shtrikman bound) or rough estimates based on model relations are available, e.g. effective medium approximations of the polynomial, power-law or exponential type [7]. In particular, this concerns the practically important case of isotropic porous materials with uniform random microstructures. Even if the microstructures are isotropic, uniform and random, the relative property estimates based on these model relations become rather unreliable as soon as the porosity becomes moderately high and the pore shape deviates from sphericity (even if the shape remains isometric on average, e.g. in the case of concave pores). Even worse is the predictability of relative properties in the case of strongly anisometric pores (including the extreme cases of 1D pore channels and 2D microcracks), even if the microstructure as a whole remains isotropic due to the random orientation of the anisometric pores.

It is clear that a universal prediction of the relative properties of porous materials based on the porosity (i.e. the pore volume fraction) alone does not exist, simply because essential microstructural information is missing and the problem is in this sense ill-posed. However, there are certain pairs of properties for which the relative values are coupled, leading either to inequalities, so-called cross-property bounds (elemental or translational) $[1,6,8,9]$, or to cross-property relations (CPRs) in the form of equations [7, 10-13]. Some of these, e.g. the Levin relation between the effective thermal expansion coefficient and the effective bulk modulus [10], are valid for arbitrary (isotropic) microstructures, whereas other, e.g. the Sevostianov-Kovacik-Simancik cross-property relation between the Young's modulus and conductivity [11], are valid only for special microstructures, e.g. the Hashin assemblage [5], see also [7]. Among the latter, our cross-property relation [14] seems to be the most realistic CPR for predicting the Young's moduli 
of isotropic porous materials with isometric pores when the conductivity is known and vice versa, because it contrast to all other CPRs it holds both for power-law materials and for exponential materials and thus can be expected to provide good approximations at least for materials which are in between these two. It is a direct consequence of the complete analogy between relations describing the porosity dependence of Young's modulus and conductivity [15]. CPRs of this type are useful e.g. in cases where the sample geometry, or the lack of instrumental equipment or numerical tools, does not allow the determination of one of these properties, while the other can be determined. Moreover, it has been shown that the mere existence of non-trivial cross-property relations of this type indicates that minimum solid area models are not useful [16]. A remarkable recent result, not yet published, is that our original CPR does not hold only for materials with convex pores and foams, but also for materials with concave pores. However, it is clear that our original CPR for isometric pores [14] cannot hold for anisometric pores. Therefore it is the purpose of this paper to derive a generalized version of our CPR that can be used for spheroidal pores of known aspect ratio. Moreover, the generalized CPR presented here does not hold only for the tensile modulus (Young's modulus), but also for other elastic moduli (shear modulus and bulk modulus), even if the Poisson ratio is not close to 0.2.

\section{THEORETICAL}

\section{Derivation of the generalized cross-property relation}

The derivation of our original cross-property relation (CPR) [14] was based on the proposition that materials that obey either a power-law or an exponential porosity dependence for one property, e.g. thermal or electrical conductivity based on Fourier's law and Ohm's law, respectively, exhibit the same type of porosity dependence also for another property, e.g. Young's modulus. Based on this proposition, our original CPR between the relative Young's modulus and the relative conductivity of isotropic porous materials with spherical or isometric pores can be written as

$$
E_{r}=k_{r}^{4 / 3},
$$

where $E_{r}=E / E_{0}$ and $k_{r}=k / k_{0}$ are the non-dimensional ratios of the effective Young's modulus and conductivity, respectively, to their counterparts for the dense solid. This CPR is based on the fact that for spherical pores the numerical coefficients of the single-inclusion solutions for conductivity (Maxwell coefficient) and Young's modulus (Dewey-Mackenzie coefficients $[17,18]$ ) are $3 / 2$ (exactly) and 2 (to an excellent approximation for solid Poisson ratios in the range $0.1-0.4$ [19]), respectively. The generalization of this CPR to other elastic moduli is straightforward, taking into account that the Dewey-
Mackenzie coefficients are functions of the Poisson ratio (of the dense solid) $v$ [19]:

$$
\begin{gathered}
{[K]=\frac{3(1-v)}{2(1-2 v),}} \\
{[G]=\frac{15(1-v)}{7-5 v},} \\
{[E]=\frac{3(1-v)(9+5 v)}{2(7-5 v)} .}
\end{gathered}
$$

Numerical values of these coefficients for the tensile, shear and bulk moduli of porous materials with spherical pores are listed e.g. in [19]. For parameterfree predictions of relative properties these coefficients have to be implemented into one of the effective medium approximations, e.g. the power-law relation [20]

$$
M_{r}=(1-\phi)^{[M]}
$$

or our exponential relation [21]

$$
M_{r}=\exp \left(\frac{-[M] \phi}{1-\phi}\right),
$$

where $M$ represents any of the moduli $E, G$ or $K$. Since completely analogous relations are available for conductivity (thermal or electrical), the generalized CPR for spherical pores (in practice a good approximation also non-spherical isometric pores) is

$$
M_{r}=k_{r}^{[M] /[k]} .
$$

Also for spheroidal pores, both oblate and prolate, the corresponding single-inclusion solutions are available [22-24] and have been extensively used [25-30]. In this case the corresponding coefficients are functions of the pore shape (aspect ratio). In particular, the Maxwell coefficients for the conductivity are given by the relation [31]

$$
[k]=\frac{3 q+2}{6 q-3 q^{2}},
$$

where for prolate pores (i.e. pores with aspect ratio larger than unity, $R=c / a>1$ )

$$
q=\frac{R\left(R \sqrt{R^{2}-1}-\operatorname{arcosh} R\right)}{\left(R^{2}-1\right)^{3 / 2}},
$$

while for oblate pores (i.e. pores with aspect ratio smaller than unity, $R=c / a<1$ )

$$
q=\frac{R\left(\arccos R-R \sqrt{1-R^{2}}\right)}{\left(1-R^{2}\right)^{3 / 2}} .
$$

Similarly, the Eshelby-Wu coeffcients for the shear and bulk moduli are functions of the aspect ratio (in addition to being functions of the Poisson ratio) [32], i.e.

$[K]=\frac{1-v}{6(1-2 v)} \cdot \frac{4(1+v)+2 R^{2}(7-2 v)-\left[3(1+4 v)+12 R^{2}(2-v)\right] \cdot q}{2 R^{2}+\left(1-4 R^{2}\right) \cdot q+\left(R^{2}-1\right)(1+v) \cdot q^{2}}$ 


$$
\begin{aligned}
& {[G]=\frac{4\left(R^{2}-1\right)(1-v)}{15\left\{8(v-1)+2 R^{2}(3-4 v)+\left[(7-8 v)-4 R^{2}(1-2 v)\right] \cdot q\right\}}} \\
& \times\left\{\begin{array}{l}
\frac{8(1-v)+2 R^{2}(3+4 v)+\left[(8 v-1)-4 R^{2}(5+2 v)\right] \cdot q+6\left(R^{2}-1\right)(1+v) \cdot q^{2}}{2 R^{2}+\left(1-4 R^{2}\right) \cdot q+\left(R^{2}-1\right)(1+v) \cdot q^{2}} \\
-3\left[\frac{8(v-1)+2 R^{2}(5-4 v)+\left[3(1-2 v)+6 R^{2}(v-1)\right] \cdot q}{-2 R^{2}+\left[(2-v)+R^{2}(1+v)\right] \cdot q}\right]
\end{array}\right\},
\end{aligned}
$$

where $R=c / a$ is the aspect ratio of the spheroidal pore and $q$ a function of the aspect ratio, see Equations 9 and 10. The Eshelby-Wu coefficient for the tensile modulus (Young's modulus) is then given by the expression [32]

$$
[E]=\frac{[K]+2[G]+2 v([G]-[K])}{3} .
$$

Figures 1 and 2 show both the Maxwell coefficients for conductivity and the Eshelby-Wu coefficients for the Young's modulus (or any other elastic modulus) in the case that the Poisson ratio (of the solid) is 0.2. It is evident that both coefficients exhibit a minimum value for spherical pore shape and asymptotically approach a limit value for prolate pores (1.667 for the Maxwell coefficient and 2.344 for the Eshelby-Wu coefficient), while for oblate pore shape both exhibit an exponential increase to infinitely high values when the aspect ratio approaches zero. While the Eshelby-Wu coefficients for the Young's modulus do not change very much with the Poisson ratio, those of the other elastic moduli, especially of the bulk modulus, are strongly dependent on the Poisson ratio [32]. Nevertheless, all the aforesaid holds qualitatively also for the other elastic moduli, only the asymptotic limit values for the prolate pores change (from 2.333, 2.667 and 1.667 for the Young's modulus, shear modulus and bulk modulus, respectively, when the Poisson ratio is 0 to $2.200,1.867$ and infinity, respectively, when the Poisson ratio is 0.49999, i.e. approaches 0.5 ).

\section{Exponents of the generalized cross-property relation in dependence of the pore aspect ratio and solid Poisson ratio}

Figures 3-5 show the values of the exponents of the generalized cross-property relation (CPR) as a function of the pore aspect ratio, with the Poisson ratio (of the solid) as a curve parameter. Tables 1-3 list the corresponding numerical values as a handy reference for the purpose of practical application. It is evident that the curves for the tensile modulus (Young's modulus) are relatively close, while those of the shear modulus exhibit a wider range of values and the range of CPR exponents for the bulk modulus is widest, becoming infinitely large as the Poisson ratio approaches 0.5 (for a Poisson ratio of 0.5 , i.e. an incompressible material, the CPR coefficient of the bulk modulus is not defined). The lowest CPR exponent (viz. a value of 1 ) is attained for the bulk modulus of a material with randomly oriented prolate pores. All other CPR coefficients are larger than

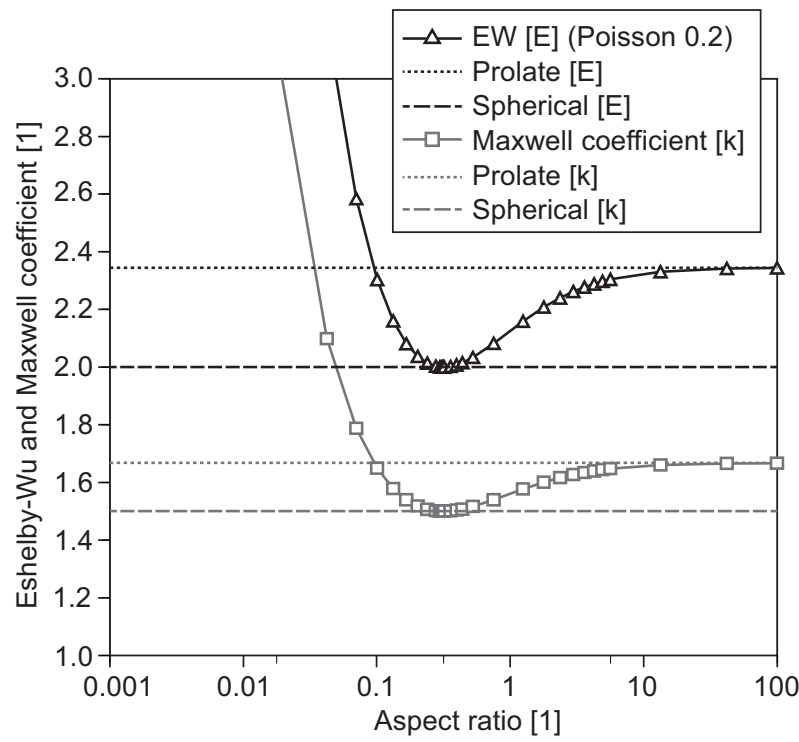

Figure 2. Maxwell and Eshelby-Wu coefficients of porous materials with spheroidal pores in dependence of the aspect ratio of spheroidal pores (semilogarithmic graph).
Figure 1. Maxwell and Eshelby-Wu coefficients of porous materials with spheroidal pores in dependence of the aspect ratio of spheroidal pores (logarithmic graph). 
unity. This agrees with the requirement that the relative bulk modulus must always be equal or smaller than the relative conductivity, i.e.

$$
K_{r} \leq k_{r}
$$

(Milton-Torquato cross-propertybound/MTbound [8,9]). In all cases the CPR exponents are limited from above and below (except for the CPR exponent for the bulk modulus of incompressible materials, which is not defined, and the CPR exponent for the bulk modulus of materials with solid Poisson ratios approaching 0.5 , for which the CPR exponent approaches infinity). All exhibit a minimum value for spherical pore shape (aspect ratio $R=1$ ) and approach finite limit values for extremely oblate and prolate pore shape. In particular, for solid Poisson ratios of 0.2 the minimum value is
$4 / 3=1.333$, see Equation 1 , the oblate $(R \rightarrow 0)$ limits are $2.001,1.704$ and 3.186 and the prolate $(R \rightarrow \infty)$ limits are $1.406,1.408$ and 1.400 for the CPR exponents of the tensile modulus (Young's modulus), shear modulus and bulk modulus, respectively, see Tables 1-3. For the tensile modulus (Young's modulus) and shear modulus minimal values of CPR exponents (1.278 and 1.111 , respectively, for spherical pore shape) are attained when the solid Poisson ratios approach 0.5 (for incompressible materials, i.e. 0.5 exactly, the CPR coefficients are not defined), while for the bulk modulus minimal values of CPR exponents (1.000 for spherical pore shape) are attained when the solid Poisson ratio is 0 . Interestingly, closer inspection reveals that the CPR exponent for the tensile modulus (Young's modulus) has a relatively complicated behavior as a function of the

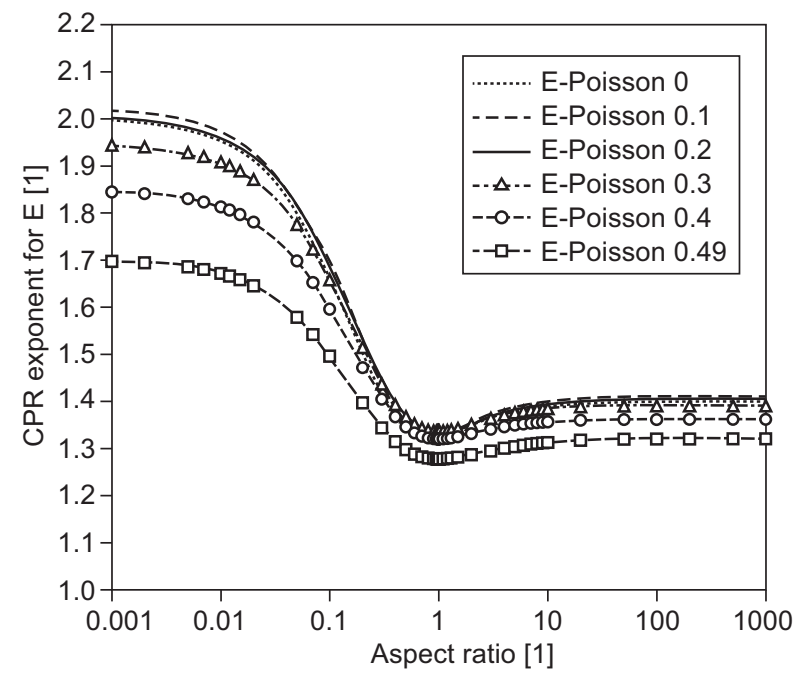

Figure 3. Cross-property relation exponent for the Young's modulus of isotropic porous materials as a function of the pore aspect ratio, with the Poisson ratio as a parameter.

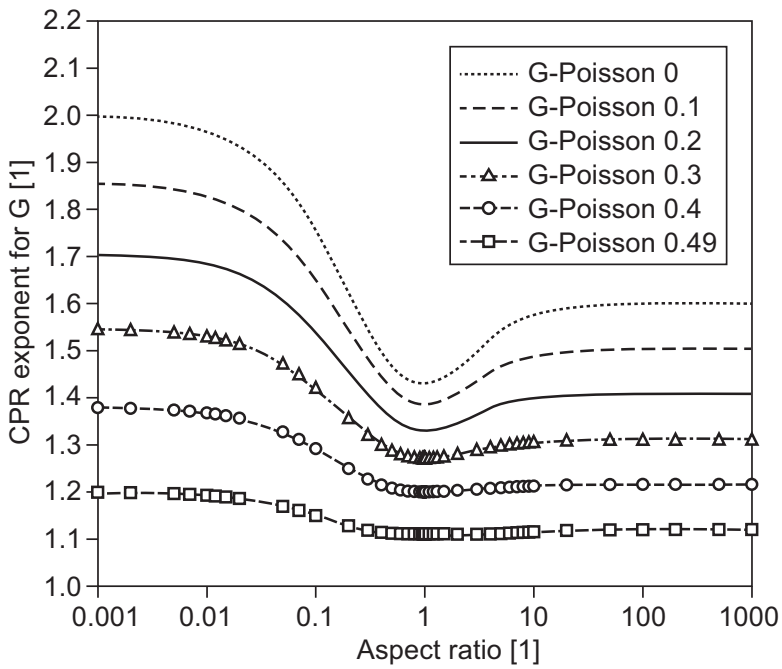

Figure 4. Cross-property relation exponent for the shear modulus of isotropic porous materials as a function of the pore aspect ratio, with the Poisson ratio as a parameter.

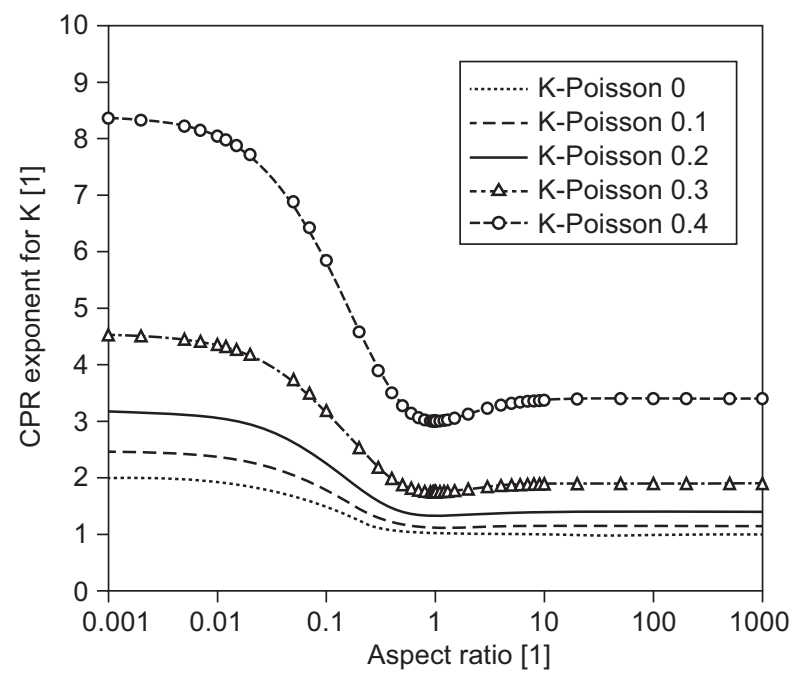

Figure 5. Cross-property relation exponent for the bulk modulus of isotropic porous materials as a function of the pore aspect ratio, with the Poisson ratio as a parameter.

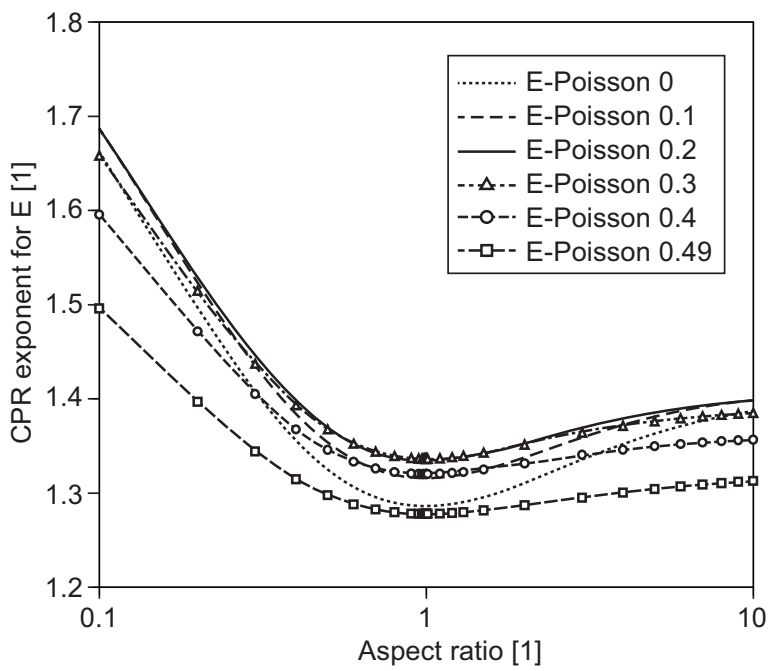

Figure 6. Cross-property relation exponent for the Young's modulus of isotropic porous materials as a function of the pore aspect ratio, with the Poisson ratio as a parameter. 


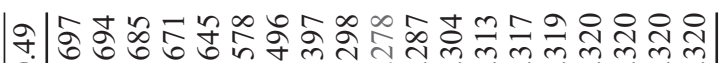
-

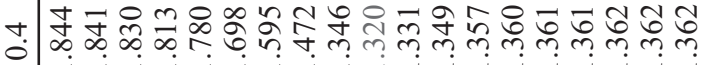

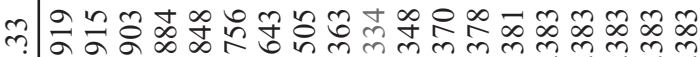
3 . $-7$

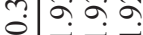

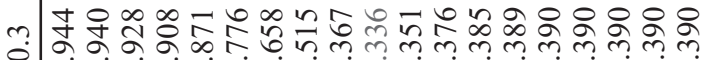

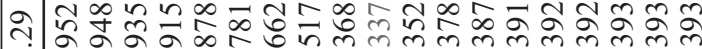
-

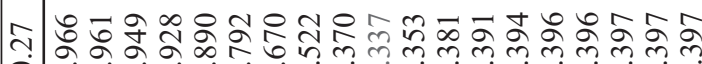

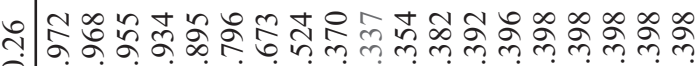

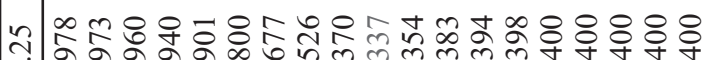

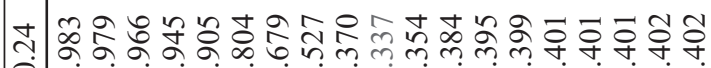

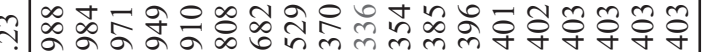

ำ

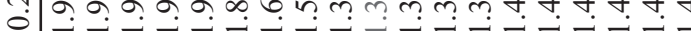

ㄱํํํำ

응ㅇㅇㅇㅇㅇ

-

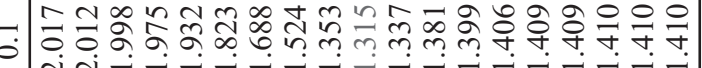

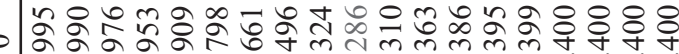

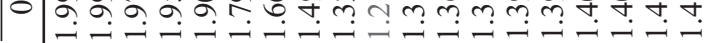

胥 $\infty$ 응
列

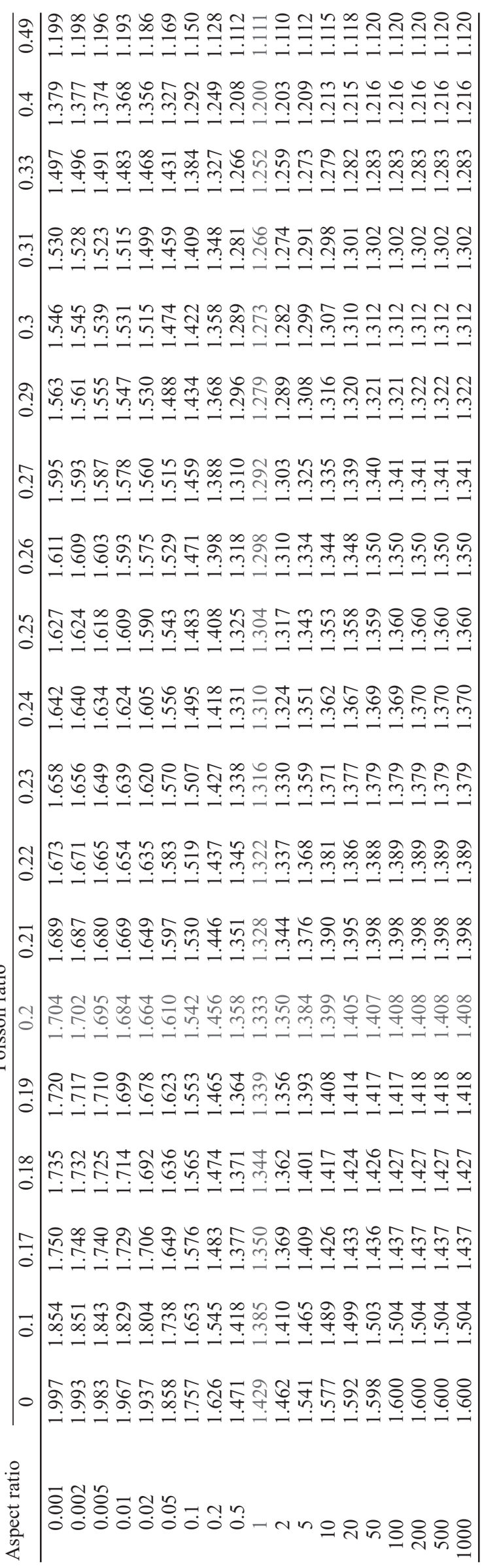

Ceramics - Silikáty 61 (1) 74-80 (2017) 


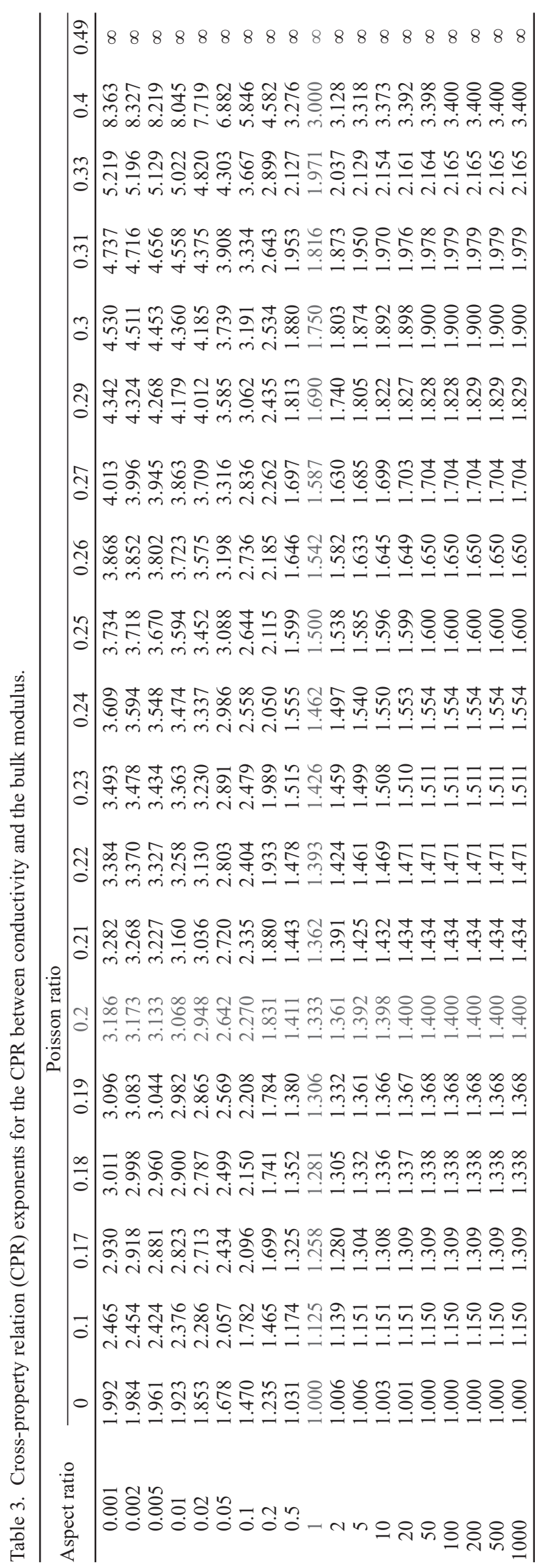

Poisson ratio: curves for different Poisson ratios can intersect each other, see Figure 6 and Table 1, so that the CPR exponents can be equal for different Poisson ratios (e.g. for spherical pores in materials with solid Poisson ratios 0.21 or 0.33 ) and thus the possibility of interpolating between different Poisson ratios is rather limited. However, the CPR exponents are very similar in this region, and thus Table 1 should be sufficient for all cases occurring in practice.

\section{SUMMARY AND CONCLUSION}

A new generalized version of our cross-property relation (CPR) between the tensile modulus (Young's modulus) and conductivity (thermal or electrical) of isotropic porous materials with isometric pores has been proposed that extends the applicability of our original CPR to other elastic moduli (shear and bulk modulus) and to randomly oriented anisometric (spheroidal) pores, including their extreme special cases, namely pore channels and microcracks. This new CPR is a handy tool to predict the relative elastic moduli when the relative conductivities are known (or vice versa) for an extremely wide range of different microstructures. Based on calculations of Maxwell coefficients and Eshelby$\mathrm{Wu}$ coefficients for prolate and oblate spheroids, the values of the CPR exponent are given in graphical and numerical (tabulated) form.

\section{Acknowledgement}

This work is part of the project "Preparation and characterization of oxide and silicate ceramics with controlled microstructure and modeling of microstructure-property relations" (GA15-18513S), supported by the Czech Science Foundation (GAČR).

\section{REFERENCES}

1. Torquato S. (2002). Random Heterogeneous Materials. Springer.

2. Green D. J. (1998). An Introduction to the Mechanical Properties of Ceramics. Cambridge University Press.

3. Gibson L. J., Ashby M. F. (1997). Cellular Solids Structure and Properties. $2^{\text {nd }}$ ed. Cambridge University Press.

4. Rice R. W. (1998). Porosity of Ceramics. Marcel Dekker.

5. Markov K., Preziosi L. (2000). Heterogeneous Media Micromechanics Modeling and Simulations. Birkhäuser.

6. Milton G. W. (2002): The Theory of Composites. Cambridge University Press.

7. Pabst W., Gregorová E. (2015): Elastic and thermal properties of porous materials - rigorous bounds and crossproperty relations (Critical assessment 18). Materials Science and Technology, 31, 1801-1808. doi:10.1080/026 
70836.2015 .1114697$.

8. Berryman J. G., Milton G. W. (1988): Microgeometry of random composites and porous media. Journal of Physics D: Applied Physics, 21, 87-94. doi:10.1088/0022-3727/ 21/1/013

9. Gibiansky L. V., Torquato S. (1996): Connection between the conductivity and bulk modulus of isotropic composite materials. Proceedings of the Royal Society London A, 452, 253-283. doi:10.1098/rspa.1996.0015

10.Levin V. M. (1967): Thermal expansion coefficients of heterogeneous materials. Mechanics of Solids, 21, 9-17.

11. Pabst W., Gregorová E. (2006): Cross-property relations between elastic and thermal properties of porous ceramics. Advances of Science and Technololgy, 45, 107-112.

12.Sevostianov I., Kováčik J., Simančík F. (2002): Correlation between elastic and electric properties for metal foams: theory and experiment. International Journal of Fracture, 114, L23-L28.

13. Sevostianov I., Kováčik J., Simančík F. (2006): Elastic and electric properties of closed-cell aluminum foams crossproperty connection. Materials Science and Engineering A, 420, 87-99. doi:10.1016/j.msea.2006.01.064

14.Pabst W., Gregorová E. (2007): A cross-property relation between the tensile modulus and the thermal conductivity of porous ceramics. Ceramics International, 33, 9-12. doi:10.1016/j.ceramint.2005.07.009

15.Pabst W., Gregorová E., Tichá G. (2007): Effective properties of suspensions, composites and porous materials. Journal of the European Ceramic Society, 27, 479-482. doi:10.1016/j.jeurceramsoc.2006.04.169

16.Pabst W. Gregorová E. (2015): Minimum solid area models for the effective properties of porous materials - a refutation. Ceramics Silikaty, 59, 244-249.

17.Dewey J. M. (1947): The elastic constants of materials loaded with non-rigid fillers. Journal of Applied Physics, 18, 578-586. doi:10.1063/1.1697691

18. Mackenzie J. K. (1950): Elastic constants of a solid containing spherical holes. Proceedings of the Physical Society London B, 63, 2-11. doi:10.1088/0370-1301/63/1/302

19.W. Pabst and E. Gregorová (2004): Effective elastic properties of alumina-zirconia composite ceramics - part 2: micromechanical modeling. Ceramics Silikaty, 48, 1423.

20.Pabst W., Gregorová E. (2003): Derivation of the simplest exponential and power-law relations for the effective tensile modulus of porous ceramics via functional equations. Journal of Materials Science Letters, 22, 1673-1675. doi:10.1023/B:JMSL.0000004645.77295.b5
21.Pabst W., Gregorová E. (2004): Mooney-type relation for the porosity dependence of the effective tensile modulus of ceramics. Journal of Materials Science, 39, 3213-3215. doi:10.1023/B:JMSC.0000025863.55048.c9

22.Polder D., van Santen J. H. (1946): The effective permeability of mixtures of solids. Physica, 12, 257-271.

23.Eshelby J. D. (1957): The determination of the elastic field of an ellipsoidal inclusion and related problems. Proceedings of the Royal Society London A, 241, 376-396. doi:10.1098/rspa.1957.0133

24. Wu T. T. (1966): The effect of inclusion shape on the elastic moduli of a two-phase material. International Journal of Solids and Structures, 2, 1-8.

25. Garboczi E. J., Douglas J. F. (1996): Intrinsic conductivity of objects having arbitrary shape and conductivity. Physical Review E, 53, 6169-6180. doi:10.1103/PhysRevE.53.6169

26.Phan-Thien N., Pham D. C. (2000): Differential multiphase models for polydispersed spheroidal inclusions: thermal conductivity and effective viscosity. International Journal of Engineering Science, 38, 73-88. doi:10.1016/S00207225(99)00016-6

27. Ordonez-Miranda J., Alvarado-Gil J. J. (2012): Effect of the pore shape on the thermal conductivity of porous media. Journal of Materials Science, 47, 6733-6740. doi:10.1007/s10853-012-6616-7

28. David E. C., Zimmerman R. W. (2011): Compressibility and shear compliance of spheroidal pores: Exact derivation via the Eshelby tensor, and asymptotic expressions in limiting cases. International Journal of Solids and Structures, 48, 680-686. doi:10.1016/j.ijsolstr.2010.11.001

29.David E. C., Zimmerman R. W. (2011): Elastic moduli of solids containing spheroidal pores. International Journal of Engineering Science, 49, 544-560. doi: 10.1016/j. ijengsci.2011.02.001

30.Garboczi E. J., Douglas J. F. (2012): Elastic moduli of composites containing a low concentration of complexshaped particles having a general property contrast with the matrix. Mechanics of Materials, 51, 53-65. doi:10.1016/ j.mechmat.2012.03.009

31.W. Pabst and E. Gregorová (2014): Conductivity of porous materials with spheroidal pores. Journal of the European Ceramic Society, 34, 2757-2766. doi:10.1016/j.jeurceramsoc. 2013.12 .040

32.W. Pabst and E. Gregorová (2014): Young's modulus of isotropic porous materials with spheroidal pores. Journal of the European Ceramic Society, 34, 3195-3207. doi:10.1016/j.jeurceramsoc.2014.04.009 